Results: Of 422 SSc patients in the database, 344 patients had SSc meeting ACR criteria. 89 patients were exclusively ATA-positive, of which $n=42$ with mild disease and $n=47$ with severe disease. Patients with severe disease appeared to be younger (mean age 50 vs $55 \mathrm{yr}$ ), more often non-caucasian (51 vs $12 \%$ ), with a longer time since non-Raynaud (median $4 \mathrm{vs} 2 \mathrm{yr}$ ) and more often diffuse skin involvement (dcSSc;62 vs $41 \%$ ), calcinosis ( 6 vs $0 \%$ ) and weight loss ( 23 vs $7 \%$ ). Stratification by disease duration, however revealed there are no real differences between mild en severe disease. Overall $47 \%$ of ATA+ patients in our cohort presented with mild SSc. When stratifying patients according to time of non-Raynaud, $55 \%$ of ATA+ patients presented with mild disease in the first disease duration quartile (median follow-up 0.5 years, range $0-0.8$ years). In the forth disease duration quartile, according non-Raynaud time (median follow-up 12.7 years, range $8.2-44.1$ ) the percentage with mild disease was still $27 \%$ (Figure 1).

Figure 1. Medsger scores of ATA patients stratified by duration non-Raynaud

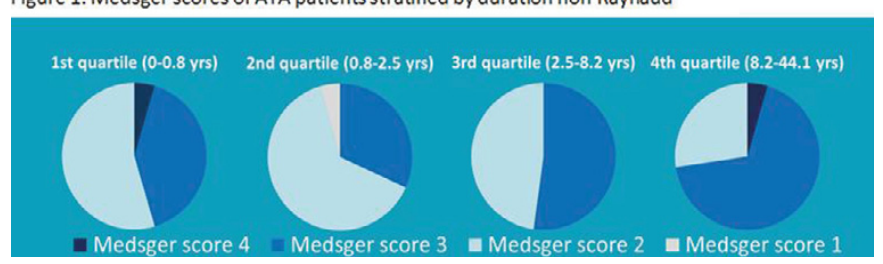

Conclusions: In our cohort, $47 \%$ of ATA-positive patients presented with mild systemic sclerosis, which could not be explained by disease duration. This suggests that solely the presence of ATA is of limited clinical relevance. Readily available sociodemographic and clinical parameters including type of skin involvement seem to have only limited value in identifying ATA patients with more severe SSc. More complex serological findings as antibody titers and fine-specifity of ATA should be defined for optimal serological subsetting.

Disclosure of Interest: None declared

DOI: 10.1136/annrheumdis-2017-eular.6414

\section{AB0651 THE EFFECT OF CYCLOPHOSPHAMIDE ON PULMONARY FUNCTION AND DEPENDENCE ON DISEASE ACTIVITY OF INTERSTITIAL LUNG DISEASE ASSOCIATED WITH SYSTEMIC SCLEROSIS}

M. Vonk ${ }^{1}$, W. van den Hombergh ${ }^{1}$, H. Knaapen ${ }^{1}$, S. Simons ${ }^{2}$, J. Fransen ${ }^{1}$, F. van den Hoogen ${ }^{1} .{ }^{1}$ department of Rheumatology; ${ }^{2}$ department of Pulmonology, Radboud University Nijmegen, Nijmegen, Netherlands

Background: The pathogenesis of interstitial lung disease associated with systemic sclerosis (SSc-ILD) is not completely elucidated, although it is believed that chronic alveolar inflammation leads to increasing fibrosis. Treatment strategies using cyclophosphamide (CYC) have been focusing on the inflammatory pathway of SSC-ILD. We hypothesized that CYC is more effective in patients that are in the early, inflammatory phase.

Objectives: The objectives of this study are to analyze the effects of intravenously CYC pulses $\left(750 \mathrm{mg} / \mathrm{m}^{2}\right)$ on pulmonary function (FVC, DLCO) in SSc-ILD after 12,24 and 36 months, and whether this effect is dependent on the extent of ILD, the proportion of ground glass compared to fibrosis, SSc disease duration or baseline DLCO $<60 \%$.

Methods: Patients with SSc-ILD receiving CYC pulses between 2003 and 2015 were classified by the Goh (2008) criteria in either limited or extensive ILD, using HRCT at baseline independently judged by two raters. Pulmonary function tests were performed at $0,6,12,24$ and 36 months. Missing outcome data due to drop-out were replaced by last observation carried forward, except in case of death.

Results: Seventy-five patients were included, 33 with limited ILD, 42 with extensive ILD. There were no baseline differences in age, gender, SSc subtype classification, disease duration or autoantibody status. Mean FVC and DLCO were stable after 12, 24 and 36 months of follow-up (figure 1).

There was no effect in the degree of change in mean FVC and DLCO for the different effect modifiers (table 2): the extent of ILD, proportion of ground glass compared to fibrosis, short SSc disease duration or baseline DLCO $<60 \%$.

Conclusions: Mean FVC and DLCO in SSc-related ILD was stable during 36 months of follow-up after cyclophosphamide pulse therapy. The extent of ILD, proportion of ground glass, SSc disease duration and baseline DLCO $<60 \%$ did not influence the effect of CYC on pulmonary function. CYC did not appeare to be more effective in the early phase of SSc-ILD.

Disclosure of Interest: None declared

DOI: 10.1136/annrheumdis-2017-eular.3441

\section{AB0652 EARLY ORGAN INVOLVEMENT, SURVIVAL AND CAUSES OF DEATH IN AN UNSELECTED COHORT OF DUTCH PATIENTS WITH SYSTEMIC SCLEROSIS}

M. Vonk, W. van den Hombergh, H. Knaapen, F. van den Hoogen, J. Fransen. Department of Rheumatology, Radboud University Nijmegen, Nijmegen, Netherlands

Background: Systemic sclerosis (SSc) patients are at risk for organ involvement and premature death. The occurrence of organ involvement that is reported differs widely between various long term cohort studies; ILD $25-90 \%$, PAH $8-32 \%, \mathrm{Cl}$ $5-30 \%$, and SRC $4-12 \%$. Differences in findings also apply to survival, the 5and 10 -year survival rates between studies vary from $80 \%$ to $90 \%$ and from $60 \%$ to $85 \%$ respectively $(1-3)$.

Objectives: To assess the occurrence of organ involvement and death in a large, unselected cohort of Dutch SSc patients at the moment of diagnosis and during 5 years of follow-up, stratified by disease subtype and auto-antibodies.

Methods: Up to 2015, 690 SSc patients were included in the Nijmegen SSc cohort. Occurrence of interstitial lung disease (ILD), pulmonary arterial hypertension (PAH), cardiac involvement (Cl), scleroderma renal crisis (SRC) and occurrence of death were determined using survival analysis, stratified by disease subtype (limited cutaneous SSc and diffuse cutaneous SSc) and auto-antibodies (ACA, ATA, anti-RNP).

Results: Organ involvement was already present at SSc diagnosis in $32 \%$ of patients. In $25 \%$, organ involvement developed during follow-up, mostly ILD (22\%). Significant differences between IcSSc and dcSSc were found in SRC at baseline and ILD, PAH and SRC during follow-up. Between the autoantibody subgroups, the occurrence of ILD, PAH and SRC at baseline and ILD during follow-up differed. There were no differences in survival between subtypes and auto-antibodies. The overall 5 -year survival rate was $89 \%$. Patients without organ involvement at SSc diagnosis had a better 5 -year survival rate than patients with organ involvement at SSc diagnosis: $95 \%$ versus $73 \%$ respectively $(p<0.001)$. (figure 1$)$

Conclusions: In many SSc patients, organ involvement is already present at diagnosis or develops in the first 5 years after diagnosis.

Survival is significantly worse in patients who already have involvement at the moment of SSc diagnosis.

\section{References:}

[1] Muangchan C, Canadian Scleroderma Research G, Baron M, Pope J. The $15 \%$ rule in scleroderma: the frequency of severe organ complications in systemic sclerosis. A systematic review. The Journal of rheumatology. 2013;40(9):1545-56.

[2] Vonk MC, Broers BM, Heijdra YF, Ton E, Snijders R, van Dijk AP, et al. Systemic sclerosis and its pulmonary complications in the Netherlands An epidemiological study. AnnRheumDis. 2008.

[3] Nihtyanova SI, Schreiber BE, Ong VH, Rosenberg D, Moinzadeh P, Coghlan $\mathrm{JG}$, et al. Prediction of pulmonary complications and long-term survival in systemic sclerosis. Arthritis \& rheumatology. 2014;66(6):1625-35.

Disclosure of Interest: None declared

DOI: 10.1136/annrheumdis-2017-eular.3426

\section{AB0653 EFFICACY OF AN INTENSIVE 24-WEEK PHYSIOTHERAPY PROGRAMME IN MYOSITIS PATIENTS - PRELIMINARY DATA FROM A SINGLE-CENTER CONTROLLED STUDY}

M. Spiritovic ${ }^{1,2}$, S. Oreska ${ }^{1}$, H. Storkanova ${ }^{1}$, P. Cesak ${ }^{2}$, A. Rathouska ${ }^{1}$ K. Kubinova $^{1}$, M. Klein ${ }^{1}$, L. Vernerova ${ }^{1}$, O. Ruzickova ${ }^{1}$, R. Becvar ${ }^{1}$, H. Mann ${ }^{1}$, K. Pavelka ${ }^{1}$, L. Senolt ${ }^{1}$, J. Vencovsky ${ }^{1}$, M. Tomcik ${ }^{1} .{ }^{1}$ Department of Rheumatology, 1st Medical Faculty, Charles University, Institute of Rheumatology; ${ }^{2}$ Faculty of Physical Education and Sport, Charles University, Prague, Czech Republic

Background: Involvement of musculoskeletal system (inflammatation, atrophy and permanent damage to the muscle) in idiopathic inflammatory myopathies (IIM) leads to impaired function and reduced muscle strength, endurance, aerobic capacity and decreased quality of life. Data on efficacy of non-pharmacologic care in IIM is very limited due to variety in studied interventions/outcomes.

Objectives: To address the limitations of existing studies, and evaluate the effect of a controlled, long-term (24-week intervention, 24-week follow-up), intensive (1h physiotherapy twice weekly, and home-exercise for $1 \mathrm{~h} 5 \mathrm{x}$ weekly), tailored physiotherapy program to improve muscle strength, endurance and deep stabilizer system, and quality of life/disability in cohorts with a substantial number of IIM patients.

Methods: All patients fulfilled the Bohan and Peter 1975 diagnostic criteria for dermatomyositis (DM) or polymyositis (PM), had skeletal muscle involvement, and were consecutively recruited from 2014 to 2016 at the Institute of Rheumatology in Prague. Both groups received educational materials and instructions for home exercise at baseline, however, only intervention group underwent the intensive physiotherapy programme. At months $0,3,6,12$ all patients were assessed by a physician [physical examination, Myositis intention to treat index (MITAX), Myositis disease activity assessment visual analogue scale (MYOACT), and Myositis damage index (MDI)], and a physiotherapist blinded to intervention [standardized tests evaluating the level of muscle strength [Manual muscle test-8 (MMT-8)], and endurance [Functional index-2 (FI-2)], patients filled out patient reported outcomes (PRO)/questionnaires [HAQ, SF-36, Beck's depression inventory-II 
(BDI-II), PROs assessing nutrition and fatigue], body composition was analyzed using densitometry (iDXA Lunar) and bioelectric impedance (BIA2000-M), and patients provided blood for routine laboratory analysis and biobanking. Normality of data was tested and inter-group analysis performed with 2-way ANOVA and intra-group analysis by Friedmann's test with Dunn's post hoc test.

Results: 27 IIM patients (22 female/5 male, 10 DM/12 PM/5 IMNM (immune mediated necrotizing myopathy), median of age 58.0 and disease duration 7.0 years) were recruited into the intervention group (IG) and 27 patients into the control group (CG) (24 female/3 male, 13 DM/12 PM/2 IMNM, median of age 56.5 and disease duration 4.7 years). Compared to observed statistically significant deterioration in $C G$ over the period of months $0-6$, we found statistically significant improvement in FI-2, MMT8, HAQ, BDI-II (Table 1). Only numerical improvement in IG compared to numerical deterioration in CG, which has not reached statistical significance, was observed in SF-36 and fatigue PROs.

\begin{tabular}{|c|c|c|c|c|c|}
\hline \multirow{2}{*}{$\begin{array}{c}\text { Parameter } \\
\text { (unit) }\end{array}$} & \multirow{2}{*}{$\begin{array}{l}\text { Intervention group } \\
\text { Mean } \pm \mathrm{SEM}\end{array}$} & \multirow{2}{*}{$\begin{array}{l}\text { Control group } \\
\text { Mean } \pm \text { SEM }\end{array}$} & \multicolumn{2}{|c|}{ Intra-group analysis (Friedmann+Dunn) } & \multirow{2}{*}{$\begin{array}{l}\text { Inter-group } \\
\text { analysis (2WA) }\end{array}$} \\
\hline & & & Interevention gr. & & \\
\hline \multirow{3}{*}{ MMT-8 } & & $m 0: 64.2 \pm 2.3$ & $m 0-m 3: p<0.001$ & $m 0-m 3: p<0.01$ & \multirow{3}{*}{$p<0.0001$} \\
\hline & $\mathrm{m} 3: 60.7 \pm 2.4$ & $\mathrm{~m} 3: 58.4 \pm 2.0$ & $m 3-m 6: p<0.01$ & $m 3-m 6: p=N S$ & \\
\hline & $m 6: 69.1 \pm 1.9$ & $\mathrm{~m} 6: 55.3 \pm 2.0$ & $m 0-m 6: p<0.001$ & $m 0-m 6: p<0.001$ & \\
\hline \multirow{3}{*}{$\mathrm{FI}-2(\%)$} & $\mathrm{mo}: 30.0 \pm 4.4$ & $\mathrm{mo}: 40.2 \pm 5.9$ & $\mathrm{~m} 0-\mathrm{m} 3: p<0.001$ & $\mathrm{~m} 0-\mathrm{m} 3: \mathrm{p}=\mathrm{NS}$ & \multirow{3}{*}{$p<0.0001$} \\
\hline & $\mathrm{m} 3: 46.9 \pm 4.7$ & $\mathrm{~m} 3: 30.8 \pm 4.9$ & $m 3-m 6: p<0.001$ & $m 3-m 6: p=N S$ & \\
\hline & $m 6: 70.6 \pm 4.9$ & $m 6: 28.7 \pm 4.5$ & $m 0-m 6: p<0.001$ & $m 0-m 6: p<0.01$ & \\
\hline \multirow{3}{*}{ HAQ } & $\mathrm{m} 0: 0.91 \pm 0.16$ & $m 0: 1.25 \pm 0.17$ & $\mathrm{~m} 0-\mathrm{m} 3: \mathrm{p}=\mathrm{NS}$ & $m 0-m 3: p=N S$ & \multirow{3}{*}{$p=0.0012$} \\
\hline & $\mathrm{m} 3: 0.69 \pm 0.14$ & $m 3: 1.33 \pm 0.18$ & $m 3-m 6: p=N S$ & $m 3-m 6: p=N S$ & \\
\hline & $m 6: 0.56 \pm 0.11$ & $m 6: 1.27 \pm 0.19$ & $m 0-m 6: p<0.001$ & $m 0-m 6: p=N S$ & \\
\hline \multirow{3}{*}{ BDI-II } & $\mathrm{m} 0: 11.9 \pm 2.1$ & $\mathrm{~m} 0: 13.3 \pm 1.5$ & $\mathrm{~m} 0-\mathrm{m} 3: \mathrm{p}=\mathrm{NS}$ & $\mathrm{m} 0-\mathrm{m} 3: \mathrm{p}=\mathrm{NS}$ & \multirow{3}{*}{$\mathrm{p}=0.0186$} \\
\hline & & & $m 3-m 6: p=N S$ & $m 3-m 6: p=N S$ & \\
\hline & $m 6 \cdot 8.9 \pm 1.5$ & $\mathrm{~m} 6: 14.6 \pm 1.4$ & $m 0-m 6: p<0.05$ & $m 0-m 6: p=N S$ & \\
\hline
\end{tabular}

Conclusions: Our intensive 24-week physiotherapy programme led to a significant improvement in muscle strength, endurance, function and depression, which was clinically meaningful in a substantial proportion of patients.

Acknowledgements: Supported by AZV-16-33574A

Disclosure of Interest: None declared

DOI: 10.1136/annrheumdis-2017-eular.4910

\section{AB0654 AN ULTRASOUND ASSESSMENT OF THE HAND AND WRIST IN EGYPTIAN PATIENTS WITH SYSTEMIC SCLEROSIS}

M. Hassanien, S. Rashad, A. Aljohee. Rheumatology Department, assuit, Egyp

Background: Systemic sclerosis (SSc, scleroderma) is a systemic disease characterized by fibrosis, progressive vascular obliteration and the production of autoantibodies.Ultrasound (US) imaging has advantages of simplicity, low cost, noninvasiveness, real-time capability and portability compared with traditional EDX. US imaging also offers high temporal and spatial resolutions, and can potentially provide dynamic anatomical information regarding local structures and kinesiology, few studies evaluate joint and tendons involvement in SSc by US the incidence and abnormality are differs among studies

Objectives: describe and determine possible correlations between US abnormalities of hand and wrist and clinical findings in SSc Egyptian patients

Methods: Randomly selected 50 Egyptian SSc patients were enrolled in the study in outpatient clinic Rheumatology department, Assuit university hospital. Clinical examination and US evaluation (Gray Scale and Power Doppler (PD)) of the tendons and joints of hands and wrists, were performed bilaterally on both dorsal and palmar sides. Joint assessment was performed on all MCP and PIP joints as well as the wrist and tendon assessment included finger flexors and extensors, wrist extensors and flexors. The scoring for synovitis was based on the EULAR/ OMERACT scoring system

Results: Within the cohort, $100 \%$ of the patients were female; mean age and range disease duration were $40.93 \pm 11.71(20-61)$ years and $8.13 \pm 5.56$ (0.5-25)years, respectively. Modified Rodnan Skin Score mean and range were $23.17 \pm 9.14(13-43), 22$ patients were diffuse type (44\%) and 28 patients were limited type (56\%), $67 \%$ had inflammatory arthralgia as reported by patients. US examination revealed abnormalities in $76 \%$ of all cases. Synovitis was present in $74 \%$ of patients ( $22 \%$ wrist; $15 \%$ MCP/PIP, $63 \%$ both); with a grade 2 and 3 synovitis in $29 \%$ cases. The grade 2 or 3 synovitis was associated with a higher age $(p<0.05)$ and disease duration $(p<0.03)$ but not with inflammatory markers (CRP, ESR) and C3 nor with anti-CCP antibodies.

Furthermore, patients with US proven grade 2 or 3 synovitis had interstitial lung disease involvement (ILD) (74\%) and elevated systolic pulmonary artery pressure $>35 \mathrm{mmHg}$ in (46\%). Grade 1 synovitis did not correlate with tenderness or joint swelling. Synovitis using PD abnormalities were present in $20 \%$ patients, but only a $25 \%$ had concomitant clinical synovitis. Bone erosions $(>1 \mathrm{~mm})$ were observed in $15 \%$, which located at the second and third MC heads and at the styloid process of the ulna. The presence of bone erosions was associated with the presence of grade 2 or 3 synovitis. US identified tenosynovitis in $40 \%$ of patients, involving the wrist extensors in the vast majority of cases $(70 \%)$, especially of the 2nd extensor compartment

Conclusions: US examination detects significant synovitis of the hand and wrist in the absence of clinical findings, and reveals structural damage in a number of patients. The grade 2 or 3 synovitis was associated with a higher age, and ILD involvement. Tendon involvement is frequent with specific pattern of wrist extensors. Thus, US seems to be a valuable tool to identify subclinical joint manifestations in Egyptian SSc patients.
Disclosure of Interest: None declared

DOI: 10.1136/annrheumdis-2017-eular.1101

\section{AB0655 ULTRASOUND B-LINES IN THE EVALUATION OF INTERSTITIAL LUNG DISEASE IN PATIENTS WITH SYSTEMIC SCLEROSIS: CUT-OFF POINT DEFINITION FOR THE PRESENCE OF PULMONARY FIBROSIS}

M. Tardella ${ }^{1}$, M. Di Carlo ${ }^{1}$, M. Carotti ${ }^{2}$, E. Filippucci ${ }^{1}$, W. Grassi ${ }^{1}$, F. Salaffi ${ }^{1}$.

${ }^{1}$ Rheumatology Department, Università Politecnica delle Marche, Jesi;

${ }^{2}$ Radiology Department, Università Politecnica delle Marche, Ancona, Italy

Background: Although in Systemic Sclerosis (SSc) the cutaneous manifestations are the most noticeable features, lung involvement guides the disease prognosis. High resolution computed tomography (HRCT) is the mainstay for the detection of the interstitial lung disease (ILD). However, exposure to ionising radiation is a major limitation for the repetition of this examination. Over the last years lung ultrasound (US) gained an intriguing potential in the evaluation of several pulmonary condition, and in previous studies was found a significant correlation between US score and HRCT score [1]

Objectives: To establish the cut-off point of the US B-lines number for detecting the presence of SSc-ILD.

Methods: Patients with SSc-ILD underwent chest HRCT, lung US, pulmonary function test, and clinical assessment. Exclusion criteria were represented by the presence of a coexisting congestive heart failure and other lung or pleural diseases (i.e., pneumonia, pleural effusion). HRCT were scored for the presence of ILD by an experienced radiologist, in accordance with the Warrick method. US assessment was performed by a US skilled rheumatologist, blinded to the HRCT results, and included the bilateral evaluation of 14 lung intercostal spaces (LIS) [2]. In each LIS the number of B-lines was recorded and summed. To test discriminant validity we used the receiver operating characteristic (ROC) curve analysis applying a Warrick score of 7 as external criterion for the presence of SSc-ILD.

Results: Forty patients completed the study. The US B-lines number and the Warrick score confirmed an excellent correlation (Spearman's rho: 0.819 , $p<0.001)$. The ROC curve analysis revealed that the presence of 10 US B-lines is the cut-off point with the greatest positive likelihood ratio (12.52) for the presence of SSc-ILD (Table).

\begin{tabular}{lcccccccc}
\hline $\begin{array}{l}\text { US B-lines } \\
\text { number }\end{array}$ & Sensitivity & $95 \% \mathrm{Cl}$ & Specificity & $95 \% \mathrm{Cl}$ & $+\mathrm{LR}$ & $95 \% \mathrm{Cl}$ & $-\mathrm{LR}$ & $95 \% \mathrm{Cl}$ \\
\hline$\geq 2$ & 100.00 & $87.2-100.0$ & 0.00 & $0.0-24.7$ & 1.00 & & & \\
$>2$ & 100.00 & $87.2-100.0$ & 15.38 & $1.9-45.4$ & 1.18 & $0.3-4.2$ & 0.00 & \\
$>3$ & 100.00 & $87.2-100.0$ & 46.15 & $19.2-74.9$ & 1.86 & $1.0-3.3$ & 0.00 & \\
$>4$ & 100.00 & $87.2-100.0$ & 61.54 & $31.6-86.1$ & 2.60 & $1.7-4.0$ & 0.00 & \\
$>5$ & 100.00 & $87.2-100.0$ & 69.23 & $38.6-90.9$ & 3.25 & $2.3-4.7$ & 0.00 & \\
$>8$ & 100.00 & $87.2-100.0$ & 76.92 & $46.2-95.0$ & 4.33 & $3.2-5.8$ & 0.00 & \\
$>9$ & 96.30 & $81.0-99.9$ & 84.62 & $54.6-98.1$ & 6.26 & $4.9-8.0$ & 0.044 & $0.004-0.4$ \\
$>10$ & 96.30 & $81.0-99.9$ & 92.31 & $64.0-99.8$ & 12.52 & $10.5-14.9$ & 0.040 & $0.003-0.6$ \\
$>11$ & 92.59 & $75.7-99.1$ & 92.31 & $64.0-99.8$ & 12.04 & $10.0-14.6$ & 0.080 & $0.008-0.8$ \\
$>14$ & 88.89 & $70.8-97.6$ & 92.31 & $64.0-99.8$ & 11.56 & $9.4-14.2$ & 0.12 & $0.01-1.0$ \\
$>15$ & 85.19 & $66.3-95.8$ & 92.31 & $64.0-99.8$ & 11.07 & $8.9-13.8$ & 0.16 & $0.02-1.3$ \\
$>16$ & 81.48 & $61.9-93.7$ & 92.31 & $64.0-99.8$ & 10.59 & $8.3-13.4$ & 0.20 & $0.03-1.5$ \\
$>19$ & 70.37 & $49.8-86.2$ & 92.31 & $64.0-99.8$ & 9.15 & $6.8-12.2$ & 0.32 & $0.04-2.3$ \\
$>20$ & 59.26 & $38.8-77.6$ & 92.31 & $64.0-99.8$ & 7.70 & $5.4-10.9$ & 0.44 & $0.06-3.1$ \\
$>21$ & 55.56 & $35.3-74.5$ & 92.31 & $64.0-99.8$ & 7.22 & $5.0-10.5$ & 0.48 & $0.07-3.3$ \\
$>22$ & 51.85 & $31.9-71.3$ & 92.31 & $64.0-99.8$ & 6.74 & $4.5-10.0$ & 0.52 & $0.08-3.6$ \\
\hline
\end{tabular}

Conclusions: The detection of $10 \mathrm{~B}$-lines is highly predictive for the HRCT presence of SSc-ILD. In SSc patients, a lung US follow-up can optimize the employment of chest HRCT.

Disclosure of Interest: None declared

DOI: 10.1136/annrheumdis-2017-eular.6828

\section{AB0656 NAILFOLD VIDEOCAPILLAROSCOPY FEATURES OF PATIENTS WITH ANTISYNTHETASE SYNDROME}

M. Sebastiani ${ }^{1}$, V. Codullo ${ }^{2}$, A. Manfredi ${ }^{1}$, A. Selva O'Callaghan ${ }^{3}$, F. Furini $^{4}$, A. Iuliano ${ }^{5}, A$. Schwarting ${ }^{6,7}$, L. Cavagna ${ }^{2}, K$. Triantafyllias ${ }^{7}$ on behalf of NASCAR Study Group. 'Policlinico di Modena, University of Modena and Reggio Emilia, Modena; ${ }^{2}$ University of Pavia, Pavia, Italy; ${ }^{3}$ Universidad Autonoma de Barcelona, Barcelona, Spain; ${ }^{4}$ University of Ferrara, Ferrara; ${ }^{5}$ Azienda Ospedaliera San Camillo-Forlanini, Roma, Italy; ${ }^{6}$ University Mainz, Mainz; ${ }^{7}$ ACURA Rheumatology Clinics, Bad Kreuznach, Germany

Background: Antisynthetase syndrome (ASSD) is an autoimmune disease characterized by the clinical triad arthritis, myositis, and interstitial lung disease (ILD). As in inflammatory myopathies, nailfold videocapillaroscopy (NVC) alterations have been sporadically described also in ASSD patients, but no elucidating data are available.

Objectives: To investigate the possible specific NVC features of ASSD patients. Methods: Within the framework of a multicenter study, we retrospectively analyzed NVC images of ASSD patients, after excluding patients with overlap syndrome with systemic sclerosis. Two operators in a blind manner re-evaluated all 